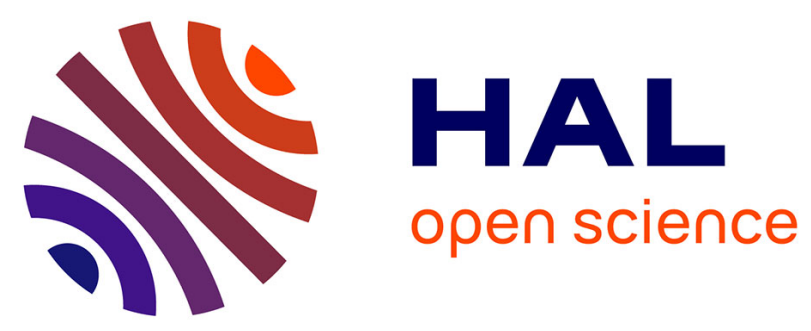

\title{
Dynamiques dynastiques au Liban: transmettre le pouvoir politique en famille
}

\author{
Ward Vloeberghs
}

\section{To cite this version:}

Ward Vloeberghs. Dynamiques dynastiques au Liban: transmettre le pouvoir politique en famille. Critique Internationale, 2016, 73, pp.71-93. 10.3917/crii.073.0071 hal-01494372

\section{HAL Id: hal-01494372 \\ https://hal.science/hal-01494372}

Submitted on 23 Mar 2017

HAL is a multi-disciplinary open access archive for the deposit and dissemination of scientific research documents, whether they are published or not. The documents may come from teaching and research institutions in France or abroad, or from public or private research centers.
L'archive ouverte pluridisciplinaire HAL, est destinée au dépôt et à la diffusion de documents scientifiques de niveau recherche, publiés ou non, émanant des établissements d'enseignement et de recherche français ou étrangers, des laboratoires publics ou privés. 


\section{Dynamiques dynastiques au Liban : transmettre le pouvoir politique en famille}

par Ward Vloeberghs

Le Liban offre un cadre exceptionnellement riche à l'analyse du phénomène dynastique en politique. On y trouve non seulement des dynasties « jeunes », dont les deux ou trois dernières générations seulement ont occupé des postes de pouvoir au niveau national, mais aussi un nombre remarquable de familles établies depuis le $\mathrm{XVIII}{ }^{\mathrm{e}}$ siècle au moins, et qui se sont transmis le pouvoir sur quatre, cinq, voire six générations.

Ces dynasties politiques prospèrent par ailleurs dans le cadre d'une démocratie consociative. Contrairement à d'autres pays de la région, le Liban ne connaît pas de régime autoritaire auquel la « dérive dynastique ${ }^{1}{ }^{1}$ servirait de mécanisme de survie. Toutefois, l'accès aux cercles du pouvoir est aujourd'hui tellement cadenassé que le renouvellement des élites politiques semble plus se faire par la promotion de la progéniture d'un ordre établi que par une compétition électorale où s’affronteraient des candidats porteurs de programmes partisans opposés.

S’il est erroné de prétendre que tout le personnel politique au Liban est issu d'une famille dynastique, on peut néanmoins affirmer que la reproduction du politique est déterminée en grande partie par l'influence persistante de ces dynasties. La notion même de "grandes familles » et leurs rapports au pouvoir politique sont bien repérés au Liban et dans la région². Selon les auteurs et les contextes, il est question tantôt de maisons (bayt pl. buyut) politiques, tantôt de familles ( 'áa'ila pl. 'à'ilāt) traditionnelles.

J'analyserai les dynamiques du phénomène dynastique (naissance, construction et consolidation) dans le système politique du Liban contemporain en mettant en contraste le

\footnotetext{
${ }^{1}$ Philippe Droz-Vincent, « Quel avenir pour l'autoritarisme dans le monde arabe ? », Revue française de science politique, 54 (6), 2004, p. 945-979. Voir également Jason Brownlee, Tariq Masoud, Andrew Reynolds, The Arab Spring: Pathways of Repression and Reform, New York, Oxford University Press, 2015 ; M. E. McMillan, Fathers and Sons. The Rise and Fall of Political Dynasty in the Middle East, Basingstoke, Palgrave Macmillan, 2013 ; Sean L. Yom, F. Gregory Gause III, « Resilient Royals: How Arab Monarchies Hang On », Journal of Democracy, 23 (4), 2012, p. 74-88 ; Michael Herb, All in the Family: Absolutism, Revolution, and Democratic Prospects in the Middle Eastern Monarchies, New York, SUNY (State University of New York) Press, 1999.

${ }^{2}$ Voir la collection d'essais sur « Les grandes familles en Méditerranée orientale » dirigée par Olivier Bouquet dans les Cahiers de la Méditerranée (82, 2011) ; Agnès Favier, "Histoires de familles, patronage et clientèles dans l'espace politique local au Liban ", Annuaire de l'Afrique du Nord, tome XLI, 2003, p. 37-66 ; Saqr Y. Saqr, 'Á'ilāt hakamat Lubnān (Ces familles qui ont gouverné le Liban), Beyrouth, al-Markaz al-'Arabī lil-ma 'alūmāt, 2008 ; Pierre Bonte, Édouard Conte, Paul Dresch (dir.), Émirs et présidents : figures de la parenté et du politique dans le monde arabe, Paris, CNRS Éditions, 2001 (nouvelle édition) (en ligne le 25 mai 2016).
} 
destin d'une maison pluriséculaire (les Salam) et celui d'une dynastie récente (les Hariri) ${ }^{3}$. Toutefois, cette comparaison de deux familles sunnites se veut avant tout une invitation à une analyse plus fine qui permettrait d'approfondir les mécanismes de transmission du pouvoir d’une génération à l'autre, au sein d’une même famille appartenant à l' « élite politiquement pertinente ${ }^{4}$, en accordant plus d'attention à la perméabilité des secteurs d'activités et aux interpénétrations entre institutions décisionnelles et structures de pouvoir politique et économique, le phénomène de concentration des connaissances, possessions et prises de décision entre les mains d’une communauté de privilégiés héréditaires n’étant pas exclusivement politique au Liban.

\section{Dynamiques dynastiques en contexte}

Cette réalité rend délicate toute tentative visant à circonscrire les dynasties politiques. La plupart de ces familles optent en effet pour une stratégie de complémentarité : certes, elles occupent des postes à responsabilité politique, mais elles sont également présentes dans les activités industrielles et commerciales du pays (Douaihy, Fadel, Hariri, Jumblatt, Tuéni). Certaines ont fait fortune grâce à l'accumulation d'un patrimoine foncier aux $\mathrm{XIX}^{\mathrm{e}}$ et $\mathrm{XX}^{\mathrm{e}}$ siècles, d'autres se sont enrichies par la spéculation immobilière aux $\mathrm{XX}^{\mathrm{e}}$ et $\mathrm{XXI}^{\mathrm{e}}$ siècles.

Si l'activité économique joue un rôle important dans la structuration du champ politique libanais, celui-ci est également quadrillé par une répartition confessionnelle: les sièges parlementaires sont attribués en vertu d'un système de quota qui diffère dans chaque circonscription selon le poids démographique de chacune des 18 communautés officiellement reconnues. Il s’ensuit que le pouvoir est principalement attribué aux (élites des) communautés confessionnelles plutôt qu'aux partis politiques, qui n’en existent pas moins pour autant ${ }^{5}$. Dans

\footnotetext{
${ }^{3}$ Au sujet de ces acteurs non traditionnels qui sont parvenus à imposer des généalogies récentes, certains évoquent l'émergence d'une nouvelle garde de représentants politiques dynastiques au Liban, comme par exemple Nicolas Sehnaoui, ministre (2011-2014) des Télécommunications, fils de l'ancien ministre Maurice Sehnaoui issu d'une famille enrichie dans l'industrie et la banque dans les années 1920-1930 ; Michel Mouawwad, fils de l'ancien président René Mouawwad et de l'ancienne députée Nayla Mouawwad, ou Ibrahim Azar, fils de l'ancien député maronite de Jezzine Samir Azar, proche de Nabih Berri. Voir Nadine el-Ali, «Political Dynasties», NOW, 8 novembre 2012 (https://now.mmedia.me/lb/en/2013elections/political_dynasties) (consulté le 10 mai 2013) ; Wassim Mroueh, « Lebanon’s Political Scions Step Up », The Daily Star, 7 février 2015.

${ }^{4}$ Le terme est emprunté à Rola El-Husseini, «Lebanon: Building Political Dynasties », dans Völker Perthes (ed.), Arab Elites. Negotiating the Politics of Change, Boulder, Lynne Rienner, 2004, p. 239266.

${ }^{5}$ Maurus Reinkowski, Sofia Saadeh, «A Nation Divided. Lebanese Confessionalism », dans Haldun 
leur gestion des conflits, les acteurs politiques ont fréquemment recours aux familles prestigieuses de ces communautés dont aucune, au demeurant, ne possède de majorité absolue. En effet, le rôle des solidarités confessionnelles est souvent surestimé. La préférence pour les familles politiques au pedigree confirmé procède à la fois d'une aspiration à la continuité de la part de ceux qui leur accordent leur confiance, et d'une disponibilité (réelle ou supposée) de ces familles en cas de besoin. Dès lors, tout type de rivalité, aussi bien locale (Zgharta contre Bcharré), historique (Yamani contre Qaysi) ou partisane (Amal contre Hezbollah) que clanique (frères contre cousins), communautaire (maronites contre orthodoxes) ou régionale (Chouf contre Hasbaya) peut servir de levier d'opposition. Ces antagonismes subtils mais profonds peuvent également constituer la base d'un rapprochement (Forces Libanaises et CPL).

L'estime pour l'institution familiale provient également d’une structure sociétale marquée par les rapports de pouvoir hérités de l'époque ottomane, lorsque la collecte de l’impôt au nom du sultan incombait aux muqāta 'jī dans les zones rurales et aux zu'amā en milieu urbain. En s’appuyant sur leur proximité avec le prince, certaines familles de notables $\left(a^{c} y \bar{a} n\right)^{6}$ ont su convertir leur puissance socioéconomique ainsi que les ressources attachées à leur fonction en pouvoir politique. D’autres n’ont pas hésité à transformer leur domination historique en une « économie morale du don », par des pratiques évergétiques, la redistribution de prébendes ou l'éthique du bienfait. Sur la base de ces ancrages profonds, Agnès Favier a dressé un profil idéal typique de la notabilité, qui comporte au moins trois dimensions : le prestige social, l'ancrage territorial et la médiation politique ${ }^{7}$. La famille et, a fortiori, l'appartenance à une « grande famille » sont ainsi des éléments structurellement déterminants dans la participation à la vie politique au $\operatorname{Liban}^{8}$.

Gülalp, Citizenship and Ethnic Conflict: Challenging the Nation-State, Londres, Routledge, 2006, p. 99-116 ; Samir Khalaf, " Primordial Ties and Politics in Lebanon », Middle Eastern Studies, 4 (3), 1968, p. 243-269; Yves Schemeil, Sociologie du système politique libanais, Grenoble, Université Pierre Mendès France, 1976 ; Myriam Catusse, Karam Karam, Returning to Political Parties? Partisan Logic and Political Transformations in the Arab World, Beyrouth, Presses de l'IFPO, 2010 ; Imad Salamey, The Government and Politics of Lebanon, Londres, Routledge, 2013.

${ }^{6}$ Albert Hourani, «Ottoman Reform and the Politics of Notables », dans Albert Hourani, William Polk, Richard Chambers (eds), The Beginning of Modernization in the Middle East: The Nineteenth Century, Chicago, Chicago University Press, 1968, p. 41-68.

${ }^{7}$ A. Favier, « Histoires de familles, patronage et clientèles dans l'espace politique local au Liban », art. cité.

${ }^{8}$ R. Hrair Dekmejian prétend que le concept de famille politique pourrait avoir plus de valeur explicative que celui de $z a$ ‘ $i m$ (titre honorifique conféré aux leaders communautaires traditionnels) : « la notion libanaise de famille etendue (' $\bar{a}$ 'ilah) pourrait s'avérer d'une utilité supérieure que le concept de $z a$ ìm pour comprendre le jeu politique et le rôle des dirigeants " (traduction WV). R. Hrair Dekmejian, Patterns of Political Leadership: Egypt, Israel, Lebanon, New York, SUNY, 1975, p. 13. Voir aussi Diane Riskedahl, «The Sovereignty of Kin: Political Discourse in Post-Ta'if Lebanon », PoLAR: Political and Legal Anthropology Review, 34 (2), 2011, p. 233-250. 
La guerre civile, qui a débuté en avril 1975 et a pris fin avec l'accord de Taëf en novembre 1989, a profondément affecté ces familles patriciennes jadis incontournables, et plusieurs d'entre elles, qui dominaient à l'époque ottomane ou mandataire des sociétés fortement hiérarchisées (par le rang social, les rapports interfamiliaux et l'accès à la propriété) ont disparu (Abillamaa, Beyhum, Chéhab, Harfoush, Nakkad, Talhouq) ou, du moins, perdu de leur influence au cours du $\mathrm{XX}^{\mathrm{e}}$ siècle ${ }^{9}$. Joseph Bahout a bien décrit les stratégies de certaines de ces familles qui, à l'issue de la guerre, ont tenté de faire bénéficier leur parentèle ou leur clientèle de la redistribution des richesses en accédant aux cercles du pouvoir ou du moins en maintenant leur relation avec ces derniers. Au niveau local, le caractère héréditaire de la transmission des responsabilités édilitaires est tenu par certaines familles pour un véritable droit ${ }^{10}$. Cependant, à l’échelle nationale, cette volonté de transmission s'est heurtée aux prétentions des seigneurs de guerre qui se sont imposés après Taëf. Si certaines grandes familles sont parvenues à survivre politiquement à la guerre, c'est parce qu'elles ont trouvé le moyen de participer aux négociations transactionelles menées par une oligarchie marchande qui s’est affirmée dans le Liban de l'après-guerre ${ }^{11}$. Cet ordre nouveau a entraîné la mainmise sur le pouvoir d'une élite restreinte (de familles établies composant avec des acteurs nettement plus récents), capable, par ailleurs, de trier sur le volet tout nouvel aspirant à une entrée sur la scène politique.

\section{Diversités confessionnelle et spacio-temporelle}

Dans le Liban d'aujourd'hui, les dynasties politiques existent dans toutes les communautés confessionnelles majeures: Karami, Jisr, Fatfat (sunnites), Chamoun, Eddé, Gemayel (maronites), Arslan, Jumblatt, Daoud (druzes), Ghosn, Murr, Sarraf (grecs-orthodoxes), Hamadé, Osseiran, Zein (chiites), Sehnaoui, Skaff, Pharaoun (grecs-catholiques). Le phénomène dynastique est donc transconfessionnel. C’est également une donnée sociopolitique qui couvre tout le territoire : les Gemayel du Metn, les Eddé du Kesrouan, les Franjieh de

\footnotetext{
${ }^{9}$ Sur ces dynasties disparues, voir Judith Dälken-Hamie, « La concurrence des légitimités au Liban Sud actuel à l'exemple des familles de zu'ama traditionnels el-Zein et el-Assaad », mémoire en politique comparée, IEP Paris, 2009 ; S. Y. Saqr, 'Á'ilāt hakamat Lubnān (Ces familles qui ont gouverné le Liban), op. cit.

${ }^{10}$ Joseph Bahout, Chaouqi Douayhi (dir.), La vie publique au Liban, expressions et recompositions du politique, Beyrouth, CERMOC, 1997.

${ }^{11}$ Sur cette oligarchie marchande, voir Fawwaz Traboulsi, A History of Modern Lebanon, Londres, Pluto, 2012 ( $2^{\mathrm{e}}$ édition) ; R. El-Husseini, Pax Syriana. Elite Politics in Postwar Lebanon, Syracuse/New York, Syracuse University Press, 2012 ; Kamal Dib, Warlords and Merchants. The Lebanese Business and Political Establishment, Reading, Ithaca Press, 2006.
} 
Zgharta, les Assaad de Marjayoun, les Khalil de Tyre, les Osseiran de Zahrani et les Hamadé de la Bekaa.

Certaines de ces maisons ont mieux réussi que d'autres à s’adapter aux évolutions politiques et socioéconomiques de leur pays. C’est le cas des Jumblatt, probablement la plus célèbre des dynasties politiques libanaises - Bechir (1775-1825), Saïd (mort en 1861), Fouad (1885-1921), Kamal (1917-1977), Walid (1949), Taymour, fils aîné de Walid (1982) -, mais aussi des Gemayel - Pierre (1905-1984), Amine (1941) et Bachir (1947-1982), Pierre (1972-2006) et Sami (1980) fils d’Amine, Nadim (1982) fils de Bachir -, des Karami - Abdel Hamid (18931950), Rachid (1921-1977) et Omar (1935-2015), Faysal (1971) fils de Omar -, des Franjieh - Qabalan (1872-1941), Hamid (1907-1981) et Suleiman (1910-1992), Samir (1945) fils de Hamid et Tony (1941-1978) fils de Suleiman, Suleiman (1964) fils de Tony, Tony (1987) fils de Suleiman ${ }^{12}$-, ou des Murr - Michel (1932), Elias (1962). A chacune de ces générations, ces familles ont livré des ministres au Liban. Elles prouvent ainsi que les dynasties ne sont pas liées à une époque particulière de l’histoire politique du pays.

Phénomène rare mais notable dans une société patriarcale, les femmes succèdent - quoique souvent de manière temporaire - à leur père (Leila Solh, Nayla Tuéni, Tracy Chamoun), à leur frère (Bahia Hariri) ou à leur époux (Nazira Jumblatt, Sethrida Geagea, Nayla Mouawwad, Solange Gemayel). Plus rare encore est la participation aux affaires de deux membres d'une même famille politiquement opposés (les cousins Faysal et Ahmad Karami au sein du cabinet Miqati (2011-2013), les cousins Mohamed et Nohad Machnouq au sein de l'actuel cabinet Salam).

\section{L'enjeu du pouvoir et le pouvoir comme enjeu}

Si le phénomène dynastique n'est pas l'apanage d'un seul parti politique, certains partis reposent plus que d'autres sur des dynasties. Ainsi, les Kataëb, les Marada, le Parti socialiste progressiste et le Mustaqbal sont respectivement les bastions des familles Gemayel, Franjieh, Jumblatt et Hariri. Quant à Michel Aoun (sans ancêtre dynastique et sans descendant masculin), il monopolise les positions clés au sein de son parti, Courant patriotique libre (CPL), au profit de ses proches : sa fille aînée, Mireille (mariée à Roy Hachem, directeur de la chaîne télévisée OTV), a remplacé sa fille Claudine (mariée à Chamel Roukoz, haut gradé de l’armée) comme directrice de son cabinet, alors que le mari de sa fille Chantale, Gebran Bassil (ministre des

\footnotetext{
12 Information International s.a.l., «Political Inheritance. Zu'ama and Sects », The Monthly, 160, novembre 2015, p. 4-21.
} 
Affaires étrangères), a été nommé chef du parti en août 2015 au détriment de Alain Aoun, neveu de Michel et député de Baabda. D’autres parlementaires CPL, comme Gilberte Zouein ou Walid Khoury, représentent chacun la troisième génération de leurs « jeunes » dynasties politiques respectives.

Certains partis politiques, en particulier chiites, comme le Hezbollah et Amal, et les partis arméniens ${ }^{13}$ semblent nettement moins travaillés par le phénomène de transmission héréditaire du pouvoir. Cela ne signifie pas que les histoires de succession familiale ne les affectent pas. Il est probable que l'absence de politiciens dynastiques au sein du Hezbollah soit un choix raisonné, une autre façon de pratiquer la distinction (morale, sociale, éthique) chère à l'élite religieuse chiite ${ }^{14}$. Ceci dit, si le Hezbollah est parmi les rares formations politiques où les noms de famille des élites traditionnelles sont moins fréquents, cela n’empêche pas les familles influentes sur le terrain d'avoir une influence réelle sur cette formation.

Aurélie Daher a montré que de multiples jeux d'alliances historiques et d'ingénierie électorale se sont déployés dans le nord de la Bekaa, et que des liens de parenté à différents échelons (familles, clans, branches) y affectent profondément l'adhésion ou non de l'électorat au Hezbollah. Il ressort de son étude l’image complexe d'un « parti idéologique ambitionnant un ralliement à sa cause qui transcenderait les référents claniques, familiaux et locaux ${ }^{15}$ mais qui, pour des raisons de survie politique, s’est trouvé obligé de composer avec des logiques partisanes, claniques et confessionnelles. Cet impératif de survie politique s’observe aussi chez Nabih Berri (chef du parti Amal et président du Parlement depuis 1992), qui organise la transmission de son pouvoir à travers le contrôle d'un vaste réseau de (hauts)fonctionnaires qui lui doivent leur nomination. De quoi nous interroger sur les ressources à disposition des dynasties.

Il apparaît clairement que la meilleure ressource pour fonder et maintenir un pouvoir dynastique dans le contexte libanais est un prédécesseur charismatique pourvu d'un mandat politique, de préférence exécutif et inscrit dans la durée ${ }^{16}$. Un mandat législatif est également

\footnotetext{
13 Ohannes Geukjian, « From Positive Neutrality to Partisanship: How and Why the Armenian Political Parties Took Sides in Lebanese Politics in the Post-Taif Period (1989-Present) », Middle Eastern Studies, 45(5), 2009, p. 739-767.

${ }^{14}$ Sabrina Mervin, « Charisme et distinction : l'élite religieuse chiite », dans Franck Mermier, Sabrina Mervin (dir.), Leaders et partisans au Liban, Beyrouth, Presses de l'IFPO/Paris, Karthala, 2012, p. 321351 ; Rodger Shanahan, « From Tyre to Teheran: Transnational Links amongst Lebanese Shi ‘ a Clerical Families », Journal of Shi ‘a Islamic Studies, 6 (3), 2013, p. 307-322.

${ }^{15}$ Aurélie Daher, « Le Hezbollah face aux clans et aux grandes familles de la Bekaa-Nord : les élections municipales de 2004 dans la ville de Baalbek », dans F. Mermier, S. Mervin (dir.), Leaders et partisans au Liban, op. cit. p. 421.

16 À titre d'exemple, parmi les membres du gouvernement actuel, Tammam Salam, Mohammad
} 
une ressource précieuse : entre 1920 et 1975, 26 familles ont, à elles seules, occupé un tiers des sièges au Parlement ${ }^{17}$, alors que, sur cette même période, les postes ministériels et parlementaires étaient monopolisés par 211 familles ${ }^{18}$. Aujourd'hui, au sein du Parlement (2009-2017), 36 députés sur 128, soit près d’un tiers, sont apparentés via une descendance directe (frère, sœur, fils ou petit-fils) ou indirecte mais proche (cousin, conjoint) à des personnalités politiques libanaises.

D’autres mandats de la fonction publique (ministères, forces de sécurité, entreprises étatiques) mais aussi des postes dans le secteur privé servent au maintien de ces dynasties au pouvoir. Dès lors, l'obtention et la distribution de la manne politique font l’objet de luttes acharnées. La culture consociative du système libanais et le perpétuel partage des pouvoirs qui s'ensuit facilitent, voire exigent parfois l'emprise des élites sur les ressources disponibles. Certains observateurs ont qualifié la pratique politique qui a émergé après Taëf (soit une répartition systématique des parts (muhāṣaṣa) entre les principaux acteurs) d'incitation directe au renforcement de l'ordre établi, avec tous les abus qui peuvent en découler ${ }^{19}$. Les familles politiques libanaises ont donc intérêt à manier plusieurs outils à la fois afin de pouvoir assumer durablement dépenses de statut et relations clientélistes. Reste à explorer comment cette stratégie se concrétise dans le cas des Salam et des Hariri.

Machnouq, Michel Pharaoun et Nabil de Freige sont tous fils d'anciens ministres alors que Ghazi Zeaiter et Boutros Harb sont respectivement fils et neveu d'anciens députés.

17 Dominique Lagarde, Scarlett Haddad, «Les grandes familles ", L'Express.fr (http://www.lexpress.fr/actualite/monde/proche-orient/les-grandes-familles_486731.html) (consulté le 3 octobre 2012). Un exemple remarquable de pouvoir hérité est le cas de Ghassan Moukheiber, qui est entré au Parlement en 2002, à l'issue d'une élection partielle disputée, organisée après le décès de son oncle Albert, député grec orthodoxe du Metn depuis 1950. Bien que n’ayant recueilli que $2 \%$ des suffrages, Ghassan Moukheiber s'est vu attribué le siège vacant par le Conseil constitutionnel, devenant ainsi le troisième candidat dans la lutte qui opposait l'anti-syrien Gabriel Murr (dont la victoire a été invalidée) à la pro-syrienne Myrna Murr (fille de Michel et sœur de Elias Murr). Ghassan Moukheiber a été réélu en 2005 et 2009.

${ }^{18}$ Iliya Harik, Man Yaḥkum Lubnan (Qui gouverne le Liban ?), Beyrouth, al-Nahar, 1972 et Antoine Nasri Messara, La structure sociale du Parlement libanais, 1920-1976, Beyrouth, Publications de l’Université libanaise, 1977 cités dans A. Favier, « Histoires de familles, patronage et clientèles dans l'espace politique local au Liban », art. cité, p. 40.

${ }^{19}$ Reinoud Leenders, Spoils of Truce. Corruption and State Building in Postwar Lebanon, Ithaca, Cornell University Press, 2012 ; Khalil al-Jammal, Les liens de la bureaucratie libanaise avec le monde communautaire, Paris, L’Harmattan, 2005. 
Les Salam, une dynastie de Beyrouth

Issus de trois générations successives de fils uniques (Muhammad, Abdallah, Abd al-Jalil) entre 1750 et 1830, les membres de la famille Salam figurent parmi les notables sunnites les plus influents de Beyrouth depuis 150 ans. Respectée par l'ensemble des acteurs politiques libanais, cette lignée prestigieuse impressionne tant par sa résilience que par son imbrication dans le tissu social de l'arrière-pays arabe.

\section{Quatre générations de notables}

Au cours du dernier quart du XIX ${ }^{\mathrm{e}}$ siècle, Beyrouth, jusque-là port secondaire, devient un centre politique d’importance régionale. Sur l'initiative de Ali Salam (1836-1885), riche commerçant de céréales en gros et politiquement ouvert aux tendances occidentales, la famille s'installe sur la colline de Moussaytbeh. Ali meurt jeune, à 49 ans, mais les solides bases de la puissance familiale qu'il a mises en place garantissent à Salim (1868-1938), le cadet de ses trois fils, une brillante carrière.

Salim prend le relais dès l'âge de 17 ans, et assume de multiples fonctions à la fois politiques (président de la municipalité, député de Beyrouth au Parlement ottoman) et économiques (président de la Banque agricole, membre du Tribunal de commerce). Ses prises de position pro-arabes face aux occupants ottomans, puis français font de lui l'un des plus éminents nationalistes d'avant-garde. Entreprenant, lucide et accessible, il s’impose comme le principal notable sunnite libanais à la veille de la première guerre mondiale. Son charisme lui vaut une popularité locale et le surnom d'Abou Ali (càd 'père d'Ali', d'après son fils aîné) ainsi qu'un réseau international de contacts qu'il met au service de ses ambitions ${ }^{20}$.

Salim a une nombreuse progéniture (neuf fils et trois filles), mais c'est son sixième fils Saëb (1905-2000), et non son fils aîné Ali (1889-1961), qui représente la maison aux élections législatives de 1943. La succession de Salim repose sur une concertation interne entre les fils cadets. Emmenés par l’un des frères, Malik, ceux-ci mettent en avant une logique pragmatique et méritocratique, et finissent par convaincre Ali de laisser Saëb, jugé plus qualifié et mieux connecté aux élites de l'époque, reprendre le flambeau ${ }^{21}$. Salim ayant lui-même succédé à son

\footnotetext{
${ }^{20}$ Kamal Salibi, « Beirut under the Young Turks: As Depicted in the Political Memoirs of Salim Ali Salam (1868-1938) », dans Jacques Berque, Dominique Chevallier (dir.), Les Arabes par leurs archives (XVI ${ }^{e}$-XX $X^{e}$ siècles), Paris, CNRS Éditions, 1976, p. 193-216.

${ }^{21}$ Entretien avec Oussama Salam, Beyrouth, 10 janvier 2016.
} 
père dans cet esprit et ce sans opposition de la part de ses frères aînés Abd al-Jalil et Kamil, la fratrie peut s’appuyer sur un antécédent prestigieux pour privilégier le principe du plus apte au détriment du principe de l’aîné (al-aqdar wa laysa l-akbar), sans que Ali se sente humilié.

Le parcours de Saëb confirme la clairvoyance de ses frères. C'est surtout à partir de la seconde moitié des années 1950 qu'il s’affirme en tant que leader communautaire traditionnel ( $\mathrm{za}$ '`̀m $)^{22}$ et comme un homme politique charismatique et habile. Cet opportunisme s'illustre lorsque Saëb n’hésite pas à sacrifier son soutien à l'Égypte de Nasser au profit d'un rapprochement avec l'Arabie Saoudite, alors opposée au socialisme arabe. De même, afin d'assurer sa survie politique, il s'avère prêt à se réconcilier avec son ancien rival, le président anti-nassérien Camille Chamoun ${ }^{23}$.

Premier ministre de six équipes gouvernementales sous quatre présidents successifs - Bichara Khoury (1943-1952), Camille Chamoun (1952-1958), Fouad Chéhab (1958-1964) et Suleiman Franjieh (1970-1976) -, Saëb Salam est également député beyrouthin pendant un demi-siècle, de 1943 à 1992. Rentré au Liban en 1994, après un exil de neuf ans durant la guerre civile, il lègue à son fils Tammam (né en 1945), l'aîné de ses cinq enfants (trois fils et des jumelles) une formation précieuse et un capital de soutien populaire considérable ${ }^{24}$.

En 1978, il a fait entrer Tammam dans l'administration de l'Association Maqassid (la principale organisation, côté sunnite, d'œuvres islamiques de bienfaisance) de Beyrouth pour qu'il se forme à la gestion des affaires publiques. Tammam devient le président de cette puissante institution philanthropique en 1982 et le restera jusqu'en 2000, date du décès de Saëb Salam. Cette année verra ensuite l'écrasante victoire électorale du nouvel homme fort, Rafiq Hariri. Quelques années auparavant, en 1996, Tammam a été élu pour la première fois comme candidat indépendant sur une liste parrainée par Rafiq Hariri, alors Premier ministre. En 2000, il perd son siège de député et décide de ne pas se présenter aux élections de 2005, lorsque l'assassinat de Hariri le 14 février change la donne ${ }^{25}$. Tammam ne tarde pas à déclarer son allégeance de principe à l’Alliance « indépendantiste » du 14-Mars menée par Saad Hariri et

${ }^{22}$ Élizabeth Picard, «Une sociologie historique du za'îm libanais », dans Charles Chartouni (dir.), Histoire, société et pouvoir aux Proche et Moyen-Orients. Travaux dédiés à Toufic Touma, Paris, Geuthner, 2001, p. 157-172.

${ }^{23}$ Michael Johnson, Class and Client in Beirut. The Sunni Muslim Community and the Lebanese State 1840-1985, Reading, Ithaca Press, 1986.

24 Nadim Shehadi, Saib Salam ", The Guardian (https://www.theguardian.com/news/2000/feb/01/guardianobituaries1), $1^{\text {er }}$ février 2000 (consulté le 25 juin 2016).

${ }^{25}$ Simon Haddad, « Pour qui nous avons voté », Outre-Terre, 4 (13), 2005, p. 335-354. 
opposé au Hezbollah qui mène le camp «loyaliste » du 8-Mars, favorable à l'axe DamasTéhéran.

À la suite de l'Accord de Doha (qui intervient après la prise, le 7 mai 2008, de quartiers sunnites soutenant Hariri par des milices chiites alliées au 8-Mars), Tammam devient ministre de la Culture (2008-2009) et réintègre l’hémicycle en 2009 grâce à une entente électorale avec Saad Hariri. Le 6 avril 2013, il est désigné par le Président Michel Sleiman (2008-2014) pour former un nouveau gouvernement. Bien qu’elle ait été annoncée par Walid Jumblatt (chef d'un bloc parlementaire dont le vote est crucial), cette nomination de Tammam au poste de Premier ministre n'aurait pu se faire sans l'aval de l'ancien Premier ministre Saad Hariri et l'assentiment de la cour saoudienne, patron extérieur des sunnites libanais. C’est donc dans une logique de risques calculés que ses patrons locaux et régionaux permettent à Tammam Salam d'émerger comme figure consensuelle à un moment où l'enlisement de la crise syrienne - due notamment à l'implication d'acteurs libanais dans les hostilités - aggrave l’usure du régime politique libanais. Pour les Salam, cette nomination de Tammam - quarante ans après la fin du dernier mandat de son père Saëb et treize ans après la marginalisation de la famille face à la victoire de Rafiq Hariri - est une réhabilitation au plus haut niveau. Il convient dès lors de s'interroger sur les moyens dont dispose cette famille pour inspirer confiance en temps d’instabilité et perpétuer son pouvoir politique.

\section{Les ressources}

C'est tout d'abord par le choix des alliances matrimoniales que la famille Salam développe un vaste et puissant réseau social de parentèle qui lui permet de renforcer son prestige et son statut public. Dès les origines, cette stratégie d’alliances avec les principales familles de notables du bilād ach-Chām (la Grande Syrie) se révèle déterminante dans la patrimonialisation de son pouvoir. Au milieu du XIX ${ }^{\mathrm{e}}$ siècle, Ali Salam s'allie indirectement avec la famille de Ahmad al-Agharr, le naqīb al-achraf (doyen des descendants du prophète, fonction hautement prestigieuse), en épousant Fatima Shatila, dont la mère, Atkeh Jabr, est la sœur de la femme d'al-Agharr ${ }^{26}$.

Le lien entre les deux familles se renforce à la génération suivante, puisque le fils de Ali et Fatima, Salim Salam, épouse Koulthoum Barbir, qui est la petite-fille de Ahmad al-Agharr et la fille de Omar Barbir, éminent juriste beyrouthin. Salim et Koulthoum ont de nombreux

\footnotetext{
${ }^{26}$ Samir Kassir, Histoire de Beyrouth, Paris, Fayard, 2004, p. 244 ; Anbara Salam Khalidi, Memoirs of an Early Arab Feminist. The Life and Activism of Anbara Salam Khalidi, Londres, Pluto Press, 2013, p. 5.
} 
enfants qui, tous, se marient avec les descendant.e.s de familles renommées non seulement de Beyrouth (Tabbara, Beyhum, Ghoraib, Barraj) mais aussi bien au-delà (Khalidi de Jérusalem, Mardam Bey de Damas). Ce faisant, il profite des ressources de l'arrière-pays levantin qui était pendant l'entre-deux-guerres un espace arabe de libre circulation aussi réel qu'il est aujourd'hui improbable et cloisonné.

Cette expansion territoriale de la dynastie Salam par les enfants de Salim est aussi le résultat d'une double union entre les Salam et l'éminente famille palestinienne Khalidi de Jérusalem²7. Muhammad Salam (1895-1958) a épousé Fatima Khalidi dont le frère Ahmad a épousé Anbara (1897-1986), sœur de Muhammad. Par la suite, la benjamine des enfants de Salim et Koulthoum, Racha (1922 -2004), a épousé l’historien Walid Khalidi, fils d'un premier mariage du précité Ahmad Khalidi (père, par Anbara, de l’historien Tarif). Sulafa Khalidi, la sœur de Walid, épouse Assem Salam (1924-2012), urbaniste respecté et petit-fils de Salim Salam par le fils aîné de celui-ci, Ali. L’accumulation du capital culturel des Salam s’accroît encore avec le mariage de Hala, fille de Malik, avec Clovis Maqsoud, ambassadeur de la Ligue arabe à Washington, puis aux Nations unies entre 1974 et 1990, et celui de Hayat, fille de Abdallah, avec l'universitaire André Liebich.

Quant à Malik Salam (1917-2000), le fils cadet de Salim, il élargit la portée de la dynastie en épousant Najwa Karami, fille de Abdel Hamid Karami, représentant d’une famille traditionnelle d'oulémas de Tripoli. Malik devient ainsi le beau-frère de Rachid et Omar Karami qui, en tant que politiciens sunnites de la capitale du nord, comptent parmi les rivaux de son frère Saëb Salam. La cohésion de la fratrie Salam n’en est pas entamée pour autant.

La deuxième ressource de la dynastie Salam est sa position dominante au sein de l'Association Maqassid, la principale institution musulmane d'enseignement moderne, fondée en 1878. Lorsque Salim accède à la présidence en 1909, il transforme profondément les modes de fonctionnement de l'institution, tant en matière de pédagogie que de recrutement et de collecte de fonds. En diversifiant les champs d'action de l'association et en optimisant investissements et gestion du patrimoine, il fait de ses œuvres sociales un puissant appareil de parrainage pour la base clientéliste de la famille, qui réussit à maintenir son contrôle sur ce formidable organe d'influence politique pendant pratiquement un siècle. Trois autres Salam (Muhammad, Saëb et Tammam) succèdent à Salim à la présidence de l’Association, dont les moyens considérables

\footnotetext{
${ }^{27}$ Au sujet des Khalidi, voir Philippe Bourmaud, « Des notables locaux et nationaux : les familles Abū Ġazālah et Khalidi et la profession médicale, de l'époque ottomane au mandat britannique en Palestine », Cahiers de la Méditerranée, 82, 2011, p. 267-296.
} 
sont utilisés par la famille pour répondre aux sollicitations des citoyens et de ses alliés par la distribution de services de proximité (éducation, santé, logement) ${ }^{28}$.

Le négoce en céréales du patriarche Ali rappelle à quel point les origines de la dynastie Salam reposent sur l'industrie agro-alimentaire. Son fils Salim est un négociant prospère qui diversifie ses affaires. Et le fils de Salim, Saëb, préside, au moment où il arrive au pouvoir, ce qui deviendra la Middle East Airlines qu’il a cofondée en 1945. En 1956, le frère de Saëb, Abdallah (né en 1909), récupère le siège familial au sein du conseil d'administration, il le gardera jusqu'en 198329 . De 1984 à 1991, son frère Malik (ministre de 1974 à 1975) préside le Conseil de développement et de reconstruction, organe semi-étatique. Les ressources économiques et financières des Salam proviennent également de leur statut de propriétaires fonciers à Beyrouth, même si la guerre a durement affecté leurs revenus ${ }^{30}$. Aujourd'hui encore, Hani (né en 1937), fils de Muhammad, dirige un holding de sociétés (GRC) spécialisé dans les ressources énergétiques du Golfe.

La quatrième ressource des Salam est leur tradition de modération religieuse. Cette tolérance envers les membres des communautés autres que la leur, y compris non musulmanes, se manifeste dès les débuts de la dynastie avec Ali, qui brave la réprobation de ses contemporains et place son fils Salim dans un collège chrétien. Son attitude inclusive découle d'une conviction personnelle : l'émulation suscitée par les méthodes et technologies occidentales permet des sauts qualitatifs à ceux qui veulent bien se les approprier. Salim lui-même, tout en observant la pratique religieuse, cultive cette même attitude libérale vis-à-vis de la religion et insiste pour que des lettrés non musulmans enseignent à ses propres enfants avant qu'ils aillent étudier en Europe ; il n’hésite pas à recruter des enseignants druzes ou protestants en dépit des vives oppositions des administrateurs de l'Association Maqassid ${ }^{31}$.

Cette tolérance religieuse permet à la famille Salam de projeter une image d'elle-même résolument moderniste et décomplexée, par exemple lorsque Anbara (fille de Salim) devient la première musulmane à se dévoiler en public. Elle conduit les Salam à promouvoir le projet politique et sociétal du nationalisme arabe au niveau du Liban, ce qui les rend célèbres dans les

\footnotetext{
${ }^{28}$ M. Johnson, «Factional Politics in Lebanon: The Case of the "Islamic Society of Benevolent Intentions" (al-maqâsid) in Beirut », Middle Eastern Studies, 14 (1), 1978, p. 56-75 ; Hannes Baumann, « The Ascent of Rafiq Hariri and Sunni Philantropy », dans F. Mermier, S. Mervin (dir.), Leaders et partisans au Liban, op. cit., p. 81-106.

${ }^{29}$ Entretien avec Nawaf Salam, Rabat, 7 mars 2013.

${ }^{30}$ Jakob Skovgaard-Petersen, «The Sunni Religious Scene in Beirut », Mediterranean Politics, 3 (1), 1998, p. 69-80.

${ }^{31}$ Sur les principes d'éducation de Salim, voir A. Salam Khalidi, Memoirs of an Early Arab Feminist. The Life and Activism of Anbara Salam Khalidi, op. cit., p. 130.
} 
quartiers populaires sunnites où ils peuvent dès lors mobiliser des soutiens et des miliciens tout en s'assurant de solides appuis du côté des dirigeants panarabes ${ }^{32}$.

Ces inclinations à l'indulgence ainsi que la solidarité transconfessionnelle des pères fondateurs de la dynastie, qui n’empêche pas une attention pour les affaires religieuses sunnites, devient une constante parmi leurs descendants : Saëb comme Tammam insistent beaucoup sur l'unité nationale du Liban et font des liens intercommunautaires un élément central de leur pratique politique. Ce principe d'inclusion ancré dans la tradition, et savamment réaffirmé, continue aujourd’hui encore à séduire un public assez large, surtout dans une société libanaise composée de minorités amalgamées et surtout en ces temps marqués par des radicalismes manichéens.

\section{Un capital symbolique patiemment accumulé}

Ces quatres éléments fondateurs ont permis aux Salam de se constituer un capital moral et symbolique - culture, évergétisme, engagement politique, pouvoir de médiation - qui légitime et perpétue leur transmission familiale du pouvoir. Pourtant, la pérennité de la dynastie n'est pas acquise. En effet, les Salam ne disposent plus des ressources leur permettant de faire face aux nouveaux riches devenus les maîtres du jeu politique (Hariri, Miqati, Safadi...). De fait, leur retour sur le devant de la scène est due en grande partie à la volonté de Riyad de ne plus miser sur un seul pion - Hariri - après la débâcle du 7 mai 2008.

La marge de manœuvre de l'actuel Premier ministre Tammam Salam est donc étroite et personne ne le sait mieux que lui. C'est pour cette raison qu'il ne manque pas une occasion de renforcer sa position locale et internationale. Ainsi s’est-il posé en médiateur habile, appuyé par l’Égypte, lorsqu'il a négocié en 2014 l'installation du cheikh Deryan comme mufti de la République en remplacement de son prédécesseur Qabbani, entré en conflit avec Hariri. Reste qu'en dépit des incertitudes qui planent sur une nouvelle succession générationnelle ${ }^{33}$, les Salam ont su limiter la dispersion du patrimoine familial et, contrairement à d'autres familles sunnites comme les Beyhum, les Daouq, les Nsouli, les Solh ou les Yafi, se redresser sur l’échiquier politique.

\footnotetext{
${ }^{32}$ M. Johnson, Class and Client in Beirut. The Sunni Muslim Community and the Lebanese State 18401985, op. cit.

${ }^{33}$ Tammam Salam a eu 70 ans en 2015. Plus que son fils, ce sont Nawaf (fils de Abdallah), représentant du Liban aux Nations unies depuis 2007, et Oussama, fils de Malik, pressenti comme ministre sous Miqati, qui seront probablement les futurs représentants de la famille.
} 
Les Hariri, une dynastie émergente

Depuis 1989, la dynastie Hariri domine la vie politique libanaise. Certes, cette occupation du devant la scène ne date que deux générations mais, que ce soit par des députés, des ministres, des Premiers ministres ou des chefs de l'opposition, elle se maintient de façon ininterrompue.

\section{La conquête du centre à partir de la périphérie}

Rafiq Hariri naît le $1^{\mathrm{er}}$ novembre 1944 dans une famille modeste de Saïda. Il est l'aîné d'une fratrie de trois enfants, composée d'une sœur, Bahia, et d'un frère, Walid (plus connu sous le nom de Shafiq). Son père, Bahaa Eddine, cultivateur appauvri par une succession de mauvaises récoltes dues aux intempéries, obtient de l'Association Maqassid de Saïda une bourse d'études pour son fils, fasciné par le nationalisme arabe. Après l'abandon de ses études de comptabilité à l’Université arabe de Beyrouth, Rafiq s'expatrie en Arabie Saoudite où il crée une société de construction qui se distingue en accomplissant dans des délais records l'exécution d'un chantier commandité par le roi Khalid (1975-1982).

Tout en développant ses activités d'entrepreneur, qui lui permettent de consolider la confiance acquise auprès du palais royal saoudien, il met ses atouts nouvellement acquis au service de sa ville natale, Saïda. Il y finance les études de 30000 jeunes et contribue à la réhabilitation du patrimoine urbain. Ce faisant, il élargit son emprise sur le terrain socio-politique local, tout en s’investissant, graduellement, à l'échelle nationale. Mécène volontariste, il multiplie les œuvres sociales et les interventions humanitaires, et devient une personnalité publique impliquée dans la résolution du conflit au Liban (discrètement d'abord, de plus en plus ouvertement après $1982)^{34}$.

Son modèle avoué est Riad al-Solh, grande figure de l'indépendance libanaise, lui aussi sunnite de Saïda. Après avoir œuvré à la signature de l'accord de Taëf, il vise la conquête du pouvoir exécutif à Beyrouth, conscient toutefois de l'importance de la tutelle syrienne. Dès sa nomination au poste de Premier ministre, à l'automne 1992, il entend récolter les dividendes d’une paix régionale. Il fait de la reconstruction de Beyrouth sa priorité et contrôle ce chantier à travers une société de droit privé dont il est l'actionnaire principal. Parallèlement, il diversifie ses participations dans d’autres secteurs économiques (médias, banque, BTP, télécom). Malgré

\footnotetext{
${ }^{34}$ H. Baumann, « Citizen Hariri and Neoliberal Politics in Postwar Lebanon », PhD, SOAS University of London, 2012.
} 
des heurts fréquents avec le président de la République et le président du Parlement, il dirige trois gouvernements d'affilée entre octobre 1992 et décembre 1998, date à laquelle il démissionne lorsque son crédit politique est entamé par des accusations de conflits d'intérêt ${ }^{35}$. Déterminé à revenir, il monte une campagne audacieuse et remporte une victoire retentissante aux élections de $2000^{36}$. Ce succès inégalé fait de lui le principal politicien sunnite et lui permet de prendre la tête de deux cabinets successifs. Sa démission, en octobre 2004, intervient sur fond de désaccords avec Damas autour de la prorogation du mandat présidentiel d’Émile Lahoud. Son assassinat, le 14 février 2005, conduit l’ONU à mettre en place le Tribunal spécial pour le Liban. La commémoration de cet événement nourrit un discours politique qui marquera la vie politique libanaise pendant toute la décennie qui suit ${ }^{37}$.

\section{La succession dynastique : un héritage négocié en famille}

Se pose alors la question de sa succession. L'une des personnes les plus en vue est sa sœur Bahia, née en 1952. Députée de la ville de Saïda depuis 1992, elle a accompagné son frère dès les débuts de la carrière politique de celui-ci. Elle se charge des questions socio-culturelles, s'engage en faveur de l'émancipation des femmes et représente la famille dans son fief de Saïda. Ses prises de parole après l'assassinat de son frère sont ciblées - elle accable le gouvernement Karami - mais mesurées - elle incite à la tolérance transconfessionelle -, ce qui amène certains à voir en elle une première Première ministre arabe ${ }^{38}$. Elle écarte toutefois rapidement cette perspective et entre au gouvernement Saniora comme ministre de l’Éducation de juillet 2008 à novembre 2009.

Le fils aîné de Rafiq Hariri, Bahaa (né en 1967), semble dès lors l’héritier politique naturel de son père. Personnalité flamboyante, Bahaa représente la parole de la famille dans les premiers jours qui suivent la disparition du patriarche. Le 20 avril 2005, à l’occasion du quarantième jour de deuil ( $a r b a$ 'inn), les Hariri publient un communiqué annonçant que Saad assumera « la responsabilité historique du leadership politique ${ }^{39}$. Il semble que cette décission résulte de

\footnotetext{
${ }^{35}$ Mark W. Neal, Richard Tansey, «The Dynamics of Effective Corrupt Leadership: Lessons from Rafik Hariri's Political Career in Lebanon », The Leadership Quarterly, 21, 2010, p. 33-49.

${ }^{36}$ Nicolas Nassif, «Les élections législatives de l'été 2000 », Monde arabe Maghreb Machrek, 169, 2000, p. 116-127.

${ }^{37}$ Ward Vloeberghs, « The Making of a Martyr: Forging Rafiq Hariri’s Symbolic Legacy », dans Are Knudsen, Michael Kerr (eds), Lebanon after the Cedar Revolution, Londres, Hurst, 2012, p. 163-183. 38 « Fill that Vacuum », The Economist, 23 mars 2005.

${ }^{39}$ Le texte est disponible en ligne sur http://www.rhariri.com/english.aspx?ID=3281 (consulté le 22 mars 2016). Trois anciens ministres proches de la famille (Bahije Tabbara, Samir Jisr et Fouad Saniora) y sont cités explicitement.
} 
plusieurs considérations. Assez vite, Bahaa indique sa volonté de se concentrer sur sa carrière dans les affaires. Par ailleurs, plusieurs acteurs politiques expriment leur préférence pour Saad dont on connaît le caractère affable, raisonnable et dont on sait qu'il est bien vu par le prince héritier saoudien Abdullah, roi de 2005 à 2015. Cette préférence est exprimée également par le leader druze Walid Jumblatt (très écouté par les Hariri au moment de l'assassinat) et par le Président Chirac dont les liens avec la famille Hariri datent de la fin des années 1970, lorsque Chirac dirige la Mairie de Paris. Enfin, Nazek Audi, la veuve de Rafiq Hariri, confirme son rôle de responsable des activités philanthropiques en se consacrant à la gestion de l'héritage de son mari ${ }^{40}$.

Saad Hariri est donc propulsé à la tête d'une campagne éléctorale aménagée pour faire de lui « le leader incontesté de sa communauté aux yeux de toutes les confessions » ${ }^{41}$. Malgré une victoire remporté à grands frais, il opte pour une période de rodage en tant que dirigeant du parti al-Mustaqbal et laisse le poste de Premier ministre au fidèle serviteur de son père défunt, Fouad Saniora. Ce n'est qu'après les élections de l'été 2009 (à nouveau fort onéreuses et débouchant sur un cabinet d'entente nationale) que les deux hommes échangent leurs fonctions et que Saad prend les rênes de son premier gouvernement (septembre 2009-janvier 2011).

Né à Riyad le 18 avril 1970, Saad est le dernier d'une fratrie de trois garçons issus du premier mariage de Rafiq Hariri (avec Nida Boustani, dont Hariri se sépare lorsqu’il démarre sa carrière en Arabie Saoudite). Il est très proche de Nazek (sa belle-mère) et de sa tante Bahia, mais, malgré son attachement à sa famille, c'est surtout à l'étranger que se déroule sa formation. Son père l'envoie faire ses études à Paris et à Washington avant de lui offrir un siège au conseil d’administration du groupe Saudi Oger à Riyad. C’est là qu'il s'établit avec son épouse syrosaoudienne, Lara al-‘Azm, dont il a trois enfants (deux fils, une fille).

Signe que la succession est le résultat d’une négociation délicate entre les différentes branches ( $a j b a \bar{a} b$ ) de la famille, Saad installe son cousin Nader Hariri (né en 1969), le fils aîné de Bahia, au poste de chef de cabinet. Nader agit comme conseiller personnel de Saad et l'accompagne dans toutes les rencontres importantes. Le deuxième fils de Bahia, Ahmad (né en 1982) est nommé secrétaire général du Courant du Futur (Tayyār al-Mustaqbal), parti politique fondé par Rafiq Hariri en 1992. Le fait que ce soient les fils de Bahia Hariri qui intègrent l'appareil politique de la dynastie afin d'en assurer la survie permet de supposer que Bahia a accepté de laisser sa place d’héritière en échange d'un rôle pour chacun de ses fils. En revanche, les enfants

${ }^{40}$ W. Vloeberghs, «The Hariri Political Dynasty after the Arab Spring », Mediterranean Politics, 17 (2), 2012, p. 241-248.

${ }^{41}$ S. Haddad, Pour qui nous avons voté, art. cité, p. 341. 
(deux fils et une fille) que Nazek a eus de son union avec Rafiq Hariri évitent de se prononcer en public sur des sujets politiques.

\section{Constructions locales, parrainage international}

Le pouvoir dynastique émérgent des Hariri peut s’expliquer en grande partie par la possession de ressources déjà identifiées chez les Salam (réseaux sociaux, domination du tissu associatif, intérêts économiques, modération religieuse). Sans doute n’est-ce pas une coïncidence, Hariri ayant eu le temps de s’inspirer du modèle des Salam pour surpasser ses concurrents, d'abord à Saïda, ensuite à Beyrouth. Cela dit, les deux dynasties étant apparues à des époques différentes, leur vitesse de déploiement n’est pas la même et les éléments clés de leur ascension non plus. Si Rafiq Hariri a réussi à s’imposer sur la scène libanaise, c’est parce qu'il a su exploiter la confiance de la cour saoudienne - en particulier de Fahd bin Abdul Aziz (prince héritier dès 1975, monarque de 1982 à 2005) - et ce en dépit des entrées qu’y avait aussi Saëb Salam. Travaillé patiemment, cet appui lui a permis de réduire, voire de récupérer les flux financiers que les princes saoudiens destinaient à l'Association Maqassid et, par conséquent, d'affaiblir les Salam.

En s’inféodant au principal bailleur de fonds des sunnites libanais et en y ajoutant l'amitié personnelle du protecteur historique des maronites, la France (à travers le Président Chirac), Hariri a construit une base très solide pour asseoir son influence. Fort de ces connections, il n’a pas tardé à tisser un réseau mondial de contacts influents qu’il a constamment élargi et dont il a sollicité à maintes reprises la solidarité - y compris financière, comme en témoignent les grandes conférences internationales de Paris pour le soutien au Liban (en 2001, 2002 et 2007). Ces réseaux sont structurellement plus importants chez les Hariri que chez les Salam, parce qu'ils permettent de renouveler le parrainage politique que commande Hariri et de démontrer sa capacité à mobiliser des personnes influentes ou célèbres au bénéfice du Liban. En revanche, les choix en matière d'alliances matrimoniales jouent un rôle secondaire chez les Hariri, même si l'épouse de Saad est issue d'une famille aristocratique de Damas. Aujourd'hui encore, celuici bénéficie du travail de défrichage entrepris par son père et poursuit la démarche de celui-ci en soignant ses relations au niveau international : un think tank américain notamment mène des recherches financées au nom de Rafiq Hariri ${ }^{42}$. Il continue, par ailleurs, à cultiver son statut de principal interlocuteur libanais des Saoudiens. De même que Rafiq Hariri avait pour tâche, au début de sa carrière, de distribuer au Liban l'aide financière de l’Arabie Saoudite, de même, en

\footnotetext{
${ }^{42} \mathrm{http}$ //www.atlanticcouncil.org/programs/rafik-hariri-center-for-the-middle-east/about-the-center.
} 
décembre 2013, Saad s’est vu confié par Riyad le soin de gérer un don de trois milliard de dollars pour équiper l'armée libanaise avec du matériel français ${ }^{43}$.

Deuxième outil structurant pour la mise en place du pouvoir haririen : la Fondation islamique pour la culture et l'éducation supérieure, établie à Saïda en 1979 et dirigée à partir de 1983 par la sœur de Rafiq, Bahia. Cette institution est aux Hariri ${ }^{44}$ ce que l’Association Maqassid est aux Salam. Comme son nom l'indique, elle s'attache principalement à promouvoir l'éducation de la jeunesse libanaise, via notamment l'attribution de bourses d'études, mais intervient également dans les services sociaux et dans les travaux d'infrastructure. De toute évidence, l’initiative des Hariri a fait des émules : en 2001 les Salam ont créé à leur tour la Fondation Saëb Salam pour la culture et l'éducation supérieure, présidée par Tammam. Cela n’a pas empêché les Hariri de poursuivre leur insertion politique sur le terrain en inaugurant, entre 2001 et 2008, plus de vingt institutions d’assistance sociale, pédagogique ou alimentaire ainsi que de centres de santé publique ${ }^{45}$.

Le troisième facteur du succès de la dynastie Hariri est sa force de frappe financière incomparable : les moyens déployés par Rafiq Hariri ont durablement déséquilibré la balance des rapports de force entre les dynasties. En obligeant ses concurrents à participer au changement d'échelle qu'il a imposé notamment par ses dépenses de campagnes électorales, il a fait monter les enchères de la participation politique. C'est ici que les investissements du milliardaire Hariri à la sortie de la guerre prennent tout leur sens : la reconstruction, les réformes administratives, la création d'entreprises nouvelles ou encore le (re)financement de la dette publique sont autant de voies ouvertes à l'enrichissement privé par des moyens publics. Hariri s'est impliqué dans tous les projets importants, ce qui lui a permis d'élargir son influence autant que ses bénéfices. Voulant s’assurer une garde rapprochée de technocrates complices, il a mis en place une économie politique qui passait par la création de rentes institutionelles (patrimonialisation d'agences étatiques), l’insertion dans des activités industrielles lucratives (immobilier, banque, médias) et la récupération de programmes officiels (chantiers urbains, dettes, prêts internationaux à l'État libanais). Son arrivée au pouvoir à un moment où l'État

${ }^{43}$ Ce don a été suspendu en février 2016, officiellement pour sanctionner le manque de soutien diplomatique du Liban après le saccage de l'ambassade saoudienne à Téhéran et pour protester contre les opérations militaires du Hezbollah en Syrie.

${ }^{44}$ Dénommée Fondation Hariri du vivant de Rafiq, elle est devenue la Fondation Rafiq Hariri après la mort de celui-ci (http://www.rhf.org.lb/). Voir également Hilal Khashan, «How Grantees Relate to Grantor: A Study on a Lebanese College Scholarship Foundation ", Research in Higher Education, 33 (2), 1992, p. 263-273 ; H. Baumann, « The Ascent of Rafiq Hariri and Sunni Philantropy », cité.

${ }^{45}$ Melani Cammett, Compassionate Communalism: Welfare and Sectarianism in Lebanon, Ithaca, Cornell University Press, 2014, p. 140. 
était faible a créé un environnement propice à l'affairisme et aux gains rapides dont a profité toute une génération d'hommes politiques au profil semblable (Miqati, Farès, Murr, Safadi) et leurs sattelites respectifs.

Ces innovations se sont produites dans un contexte où les féodalités familiales de l'avant-guerre avaient été mises à mal, quand elles n'avaient pas été remplacées, par les chefs de milices dominants lors de la signature de l'accord de Taëf. Dès lors, il a été relativement facile pour Hariri de se constituer un réseau d'alliés fidèles en quête de ressources, et relativement difficile pour ceux qui tentaient de lui résister de maintenir à terme leur opposition. Comme l'a écrit Emmanuel Bonne, l'empire Hariri s'est construit sur la base d'un «clientélisme haut de gamme » dans lequel le patron ne devait s'assurer que du soutien d'une élite commandant les réseaux subalternes ${ }^{46}$. C’est grâce à ce mécanisme alimenté par des ressources incommensurables (entreprises privées, finances publiques et pétrodollars saoudiens) que Hariri a pu non seulement briser la position de leaders traditionnels à Saïda (Bizri, Saad) comme à Beyrouth (Daouq, Salam), mais aussi se distinguer, entre 1992 et 2005, de n'importe quel autre acteur ou famille politique, y compris des familles aisées comme les Salam. Cette fortune étant un élément aussi important dans la structuration du pouvoir de la dynastie, on comprend que les problèmes financiers de Saad (qui ont commencé en 2006 et se sont aggravés en 2011) aient fait couler autant d'encre ${ }^{47}$.

La dernière ressource du modus operandi de la dynastie Hariri est son discours en matière de religion. Tout au long de son parcours, la famille s'est présentée comme l'incarnation de la modération du sunnisme musulman au Liban. À l'époque de Rafiq Hariri, cette posture a permis une entente avec la bourgeoisie maronite en vue de mettre fin à la guerre et d'amorcer la reconstruction. Après 2005, les héritiers de Rafiq ont profité d’une alliance pluriconfessionnelle pour légitimer Saad dans son rôle de successeur de la lignée.

La modération religieuse des Hariri est une stratégie politique consensuelle, contrairement à celle des Salam, qui résulte d’une orientation philosophique atavique. Tolérant mais déterminé, inspiré aussi par l'exemple des Salam et d'autres notables libanais, Rafiq Hariri a adopté un positionnement centriste, susceptible de séduire aussi bien les chrétiens que les musulmans, tout en conservant une marge de profilage communautaire en cas de nécessité. Reprenant la

\footnotetext{
${ }^{46}$ Emmanuel Bonne, Vie publique, patronage et clientèle. Rafic Hariri à Saïda, Beyrouth, CERMOC, 1995, p. 98.

47 Why Is Hariri Back in Lebanon?, 18 mars 2016 (http://www.almonitor.com/pulse/originals/2016/03/lebanon-saad-hariri-return-financial-crisis.html) (consulté le 25 juin 2016).
} 
consigne paternelle, Saad a pu s'ériger en défenseur d’un islam fréquentable, à l’opposé des salafistes ou des jihadistes de Daech. Reste qu'en fonction du contexte politique Rafiq comme Saad ont pu être amnés à adopter des positions nettement plus tranchées en matière confessionelle afin de séduire la « rue sunnite ${ }^{48}$.

On peut illustrer la capacité des Hariri à manipuler le registre religieux et symbolique en étudiant leur usage de l'urbanisme comme instrument de pouvoir. La « conquête » de Saïda par Rafiq a été parachevée par l'inauguration posthume d'une grande mosquée dédiée à la mémoire de son père, à l'entrée de la ville, tandis qu'une mosquée plus modeste avait déjà été élevée à la mémoire de sa mère. Dans une veine plus ambitieuse encore, Rafiq a œuvré - financièrement mais pas seulement - à la construction de la mosquée Mohammed al-Amine sur la place des Martyrs de Beyrouth, dans un style néo-ottoman symbolisant l'élargissement de l'influence des sunnites dans le Liban d'après guerre. Inachevé au moment de sa mort, cet édifice imposant domine les bâtiments voisins : la cathédrale Saint-Georges des maronites et un espace commémoratif qui abrite sa tombe ${ }^{49}$. On retrouve cette propension à circonscrire symboliquement et matériellement son territoire dans l'emplacement de la résidence palatiale de Rafiq, à Qoraytem, au cœur du quartier sunnite de Beyrouth et tout près des Salam installés dans le quartier de Mossaytbeh. Quant à Saad, il a marqué sa prise de pouvoir dynastique en s’installant dans une résidence nommée bayt al-wasaț (Center House), dans le quartier central de Beyrouth reconstruit après la guerre. Indépendamment de sa proximité avec le sérail, qui permet d'éviter les déplacements à risques, le nouveau siège du pouvoir offre l'avantage de symboliser par son nom même et son emplacement le discours de la modération (wasațiyya) politique et prône l'entente communautaire alors même que la région s'abîme dans un confessionnalisme violent. L’inconvénient de ce déménagement est en revanche son manque d’ancrage populaire puisque le nouveau siège de la dynastie ne se situe pas dans les quartiers sunnites, lesquels forment sa principale base électorale et reprochent parfois à Saad Hariri un leadership trop effacé.

\footnotetext{
${ }^{48}$ Victor Gervais, «L'ascension politique de Rafiq Hariri : ampleur et limite de l'émergence d'un leadership sunnite unifié », dans F. Mermier, S. Mervin (dir.), Leaders et partisans au Liban, op. cit., p. 107-136.

${ }^{49} \mathrm{~W}$. Vloeberghs, Architecture, Power and Religion in Lebanon. Rafiq Hariri and the Politics of Sacred Space in Beirut, Leyde, Brill, 2016.
} 


\section{Les Hariri : accumulation rapide de capital et symbolique}

L'histoire de la dynastie des Hariri est celle d'une succession construite dans l'urgence après la disparition brutale de son fondateur en 2005. Or ce qui marque cette histoire, c'est la fulgurante ascension sociale et politique de ce fondateur, « Mister Lebanon », au lendemain de la guerre civile. C’est la métamorphose accélérée d’un homme d’affaires charismatique devenu milliardaire en entrepreneur politique devenu inamovible.

Son prestige et sa fortune n’ont été transmis à son fils qu'en partie, Rafiq n’ayant pas préparé de succession dynastique. Novice en politique, Saad s'est donc retrouvé subitement au premier plan et a dû procéder par tâtonnements, même s'il a su s'entourer de conseillers avertis dont certains étaient déjà dans l'entourage de son père. La fragilité de cet arrangement est apparue à plusieurs reprises et la question est désormais de savoir si Saad saura conserver l'appui du régime saoudien, devenu plus agressif sur le plan régional depuis les règnes de Salman et de son fils Mohammed. Outre ses déboires financiers persistants, son éviction du poste de Premier ministre par le Hezbollah en 2011, son exil sécuritaire auto-imposé et l'absence d'une vision politique convaincante qui pourrait vraiment se prétendre au service de la population et non plus d'un circuit clientéliste ont affaibli sa position, comme en témoigne la pugnacité de ses adversaires - Rifi à Tripoli et Beirut Madinati à Beyrouth - lors des élections municipales du printemps 2016.

L'influence de la dynastie Hariri a suivi un parcours chaotique ces dix dernières années et sa pérennité est loin d’être acquise. Revenu au Liban en février 2016, Saad conserve toutefois de bons atouts par rapport à d'autres acteurs politiques sunnites au Liban, les Hariri faisant partie intégrante du Liban post-Taëf. Il serait dès lors prématuré de sous-estimer les ressources - politiques et financières - de Saad Hariri et de sa famille, des resources capables un jour de lui faire retrouver son poste de Premier ministre. 


\section{Conclusion}

Plusieurs facteurs entrent en jeu dans la construction et la conservation du pouvoir politique familial dans un système concurrentiel. Tout d'abord, le contexte historique de la génèse de la dynastie auquel est par ailleurs liée sa vitesse de déploiement. La consolidation au long cours des Salam, famille notabiliaire des époques ottomane et mandataire, contraste avec l'ascension vertigineuse des Hariri, nés dans un monde où tout se fait et se défait à un rythme nettement plus élevé.

Ensuite, le projet politique. L’identité politique des Salam s'est construite autour de l'idée d’un Liban indépendant, pro-arabe et progressiste, plus à l'écoute des gens de quartier. Les Hariri, eux, défendent le legs du fondateur : un programme pro-occidental et cosmopolite, favorisant la classe entrepreneuriale afin de (re)positionner Beyrouth en tant que plaque tournante bancaire et touristique au sein d'un Moyen-Orient célébrant les possibilités du capitalisme mondialisé (pour ceux qui en ont les moyens).

Par ailleurs, chez les Salam comme chez les Hariri, la transmission du pouvoir passe par une négociation interne à la famille (ou au clan), conformément à « la tradition arabe [qui] semble méconnaître la primogéniture ou quelque autre rang par ordre de naissance, et même ne retenir aucune notion de préférence pour organiser la succession politique $»^{50}$.

Enfin, la résilience apparente des dynasties politiques ne doit pas faire oublier la menace permanente de précarité. L'irruption d'une crise, la disparition d'un allié, le revirement des alliances, l'arrivée soudaine d'un concurrent ou une gestion hasardeuse des biens familiaux peuvent faire basculer le destin d'une dynastie politique.

De fait, les péripéties de ces familles témoignent de l'existence d'un système d'alternance constante sur fond de (re)arrangements locaux et régionaux permanents. Ce schéma rappelle la théorie énoncée par Ibn Khaldūn des cycles de domination et de renversement du pouvoir par la force d'un lignage commun. C’est cet «effet de compétition générale des groupes se réclamant d'ascendances agnatiques communes » qui explique la durabilité des dynasties au Liban. Bien que l'analogie avec l'esprit de corps (aṣabiyya) qui contribuait à l'édification d'un ordre politique (dawla) ne s'applique ici que partiellement (ne serait-ce que parce que l'ordre tribal qui structurait le monde rural du temps de Ibn Khaldūn est largement absent dans le Liban actuel), on peut penser à une aṣabiyya familiale au sens où l'appartenance (par le sang ou par

\footnotetext{
${ }^{50}$ P. Bonte, É. Conte, P. Dresch (dir.), Émirs et présidents : figures de la parenté et du politique dans le monde arabe, op. cit., p. 24.
} 
le clientélisme) à telle ou telle dynastie politique crée une cohésion potentiellement disruptive parmi ses membres ${ }^{51}$. Or, loin de révéler l'existence d'énergies d'élimination mutuelle systématique, l'alternance des familles dynastiques démontre que ces familles non seulement n’existent pas de manière autonome mais aussi qu'elles concluent constamment entre elles des marchés privés et des affaires publiques. Qu’elles soient anciennes ou relativement jeunes, les dynasties politiques libanaises, bien que rivales, ont donc besoin les unes des autres pour survivre. Cette interdépendance confirme qu'elles sont, plus que jamais, dynamiques, et que la notion même de famille dynastique est un concept analytiquement pertinent pour la compréhension du politique dans ce pays ${ }^{52}$.

Ward Vloeberghs est politiste et arabisant. Après des séjours d’études en Égypte et au Liban il a travaillé au Maroc (EGE Rabat, Centre Jacques Berque) et enseigne actuellement en sciences politiques à l'Erasmus University College de Rotterdam. wardvloeberghs@yahoo.com

\footnotetext{
${ }^{51}$ Ibn Khaldūn maintient par ailleurs « que le lignage contient un principe de dégénérescence, et que le prestige d'une famille s'éteint au bout de quatre générations » : «L'illustration d'une famille ne dure pas au-delà de la quatrième génération. Celui qui a été le bâtisseur de la gloire de sa famille connaît ce qu'il lui en a coûté. Il veille à conserver les qualités qui ont permis à cette gloire de naître et de durer. Son fils, qui vient après lui, a été au contact étroit avec son père, et a donc pu entendre et apprendre tout cela de lui. Mais il est inférieur à son père, pour autant que le savoir par ouï-dire soit inférieur à l'expérience directe. Le représentant de la troisième génération se contente de marcher sur les traces de ses prédécesseurs et de les imiter. Son infériorité par rapport à la deuxième génération est celle qui existe entre un imitateur et celui qui réfléchit par lui-même. La quatrième génération est, à tous égards, inférieure aux précédentes. Son représentant a perdu les qualités qui avaient permis la conservation de la gloire familiale ». Ibn Khaldūn, Muqaddima, Paris, Gallimard, 2002 (trad. Abdesselam Cheddadi), p. 392-393, cité dans O. Bouquet, «Famille, familles, grandes familles : une introduction », Cahiers de la Méditerranée, 82, 2011, p. 189-211.

${ }^{52} \mathrm{~L}$ 'auteur exprime ici sa reconnaissance aux collègues relecteurs et relectrices pour leurs commentaires et suggestions.
} 


\section{TABLES Généalogiques:}

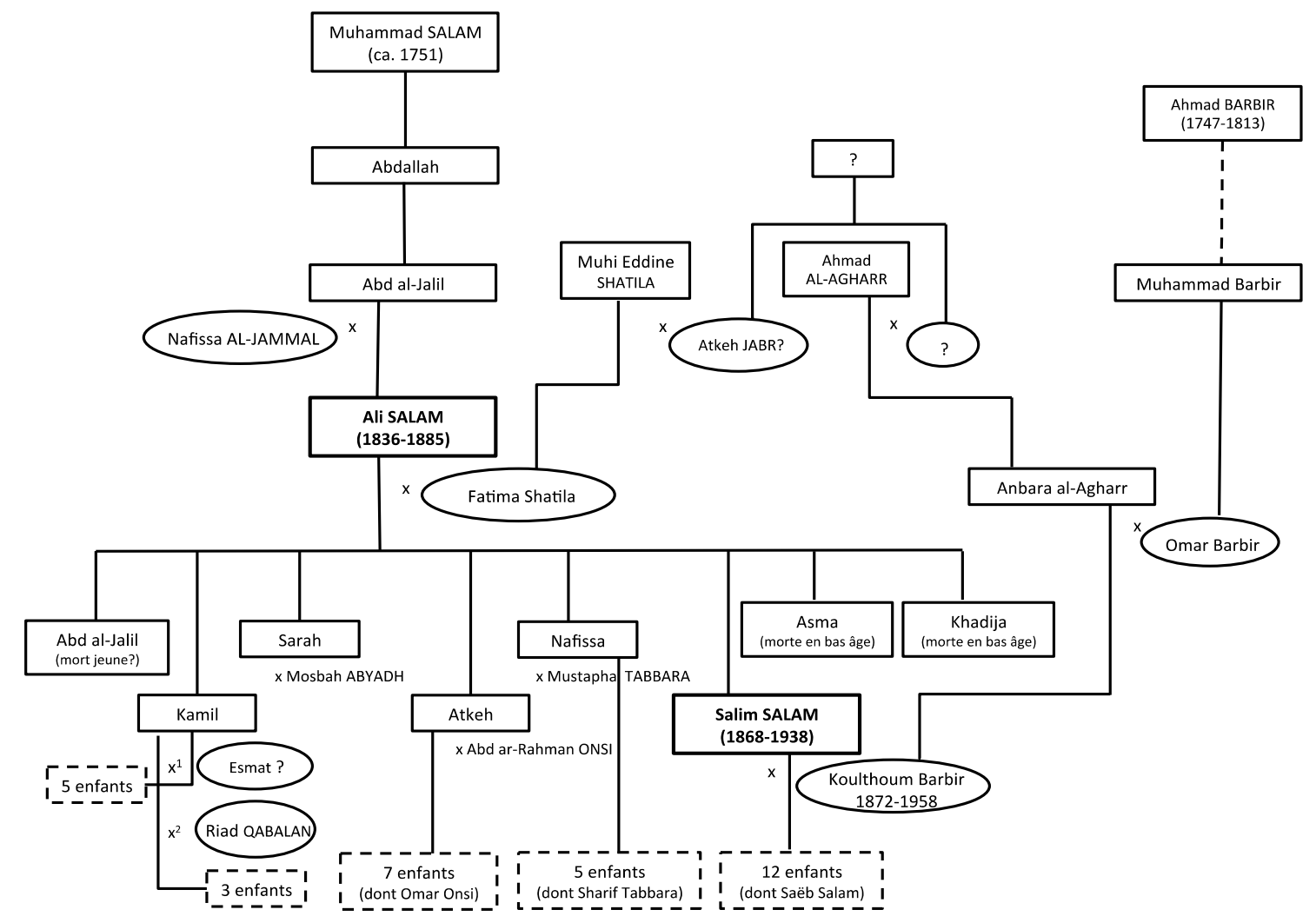




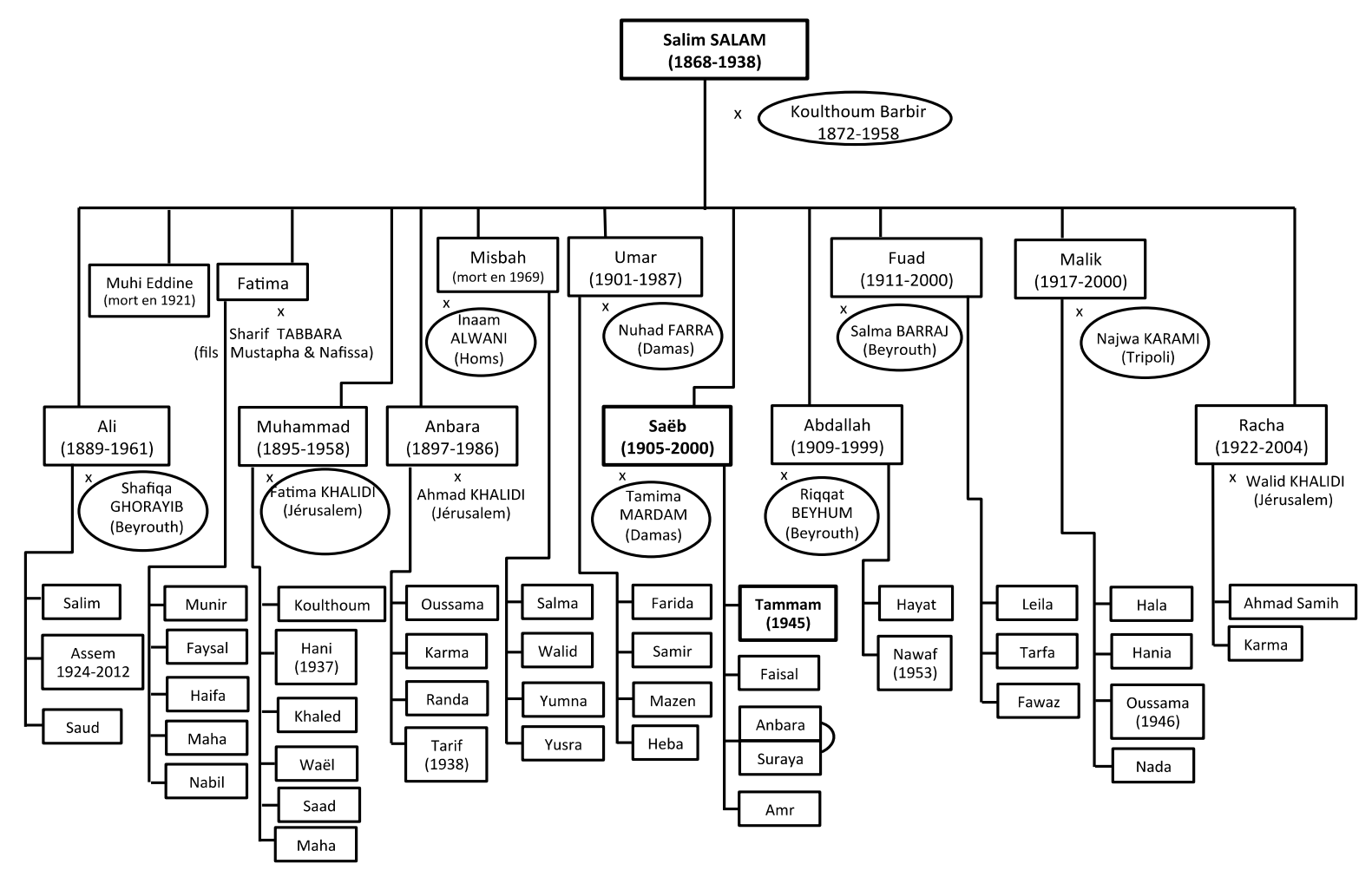




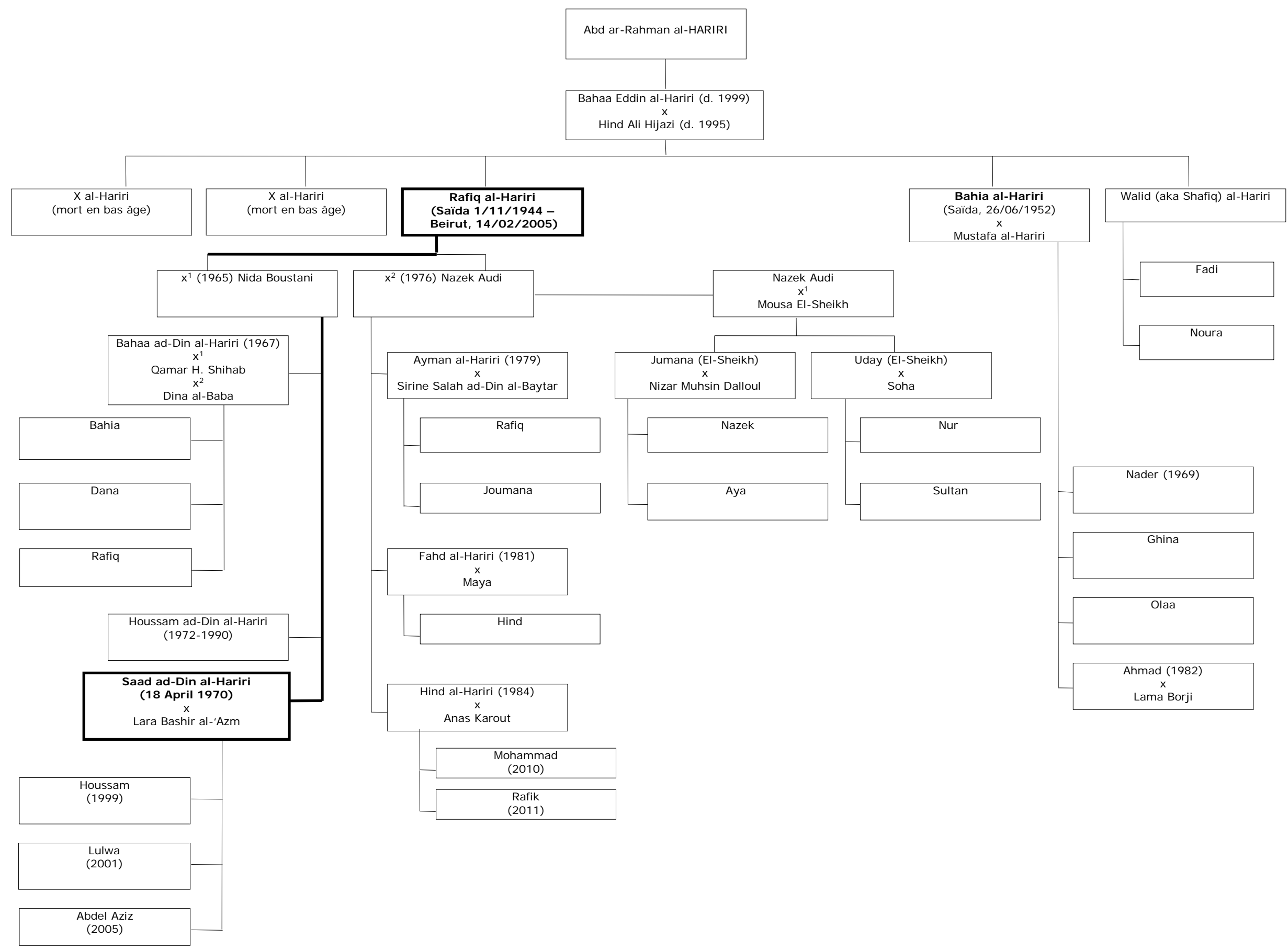

